\title{
AUFSICHTSRAT
}

\section{Kein rechtsfreier Raum}

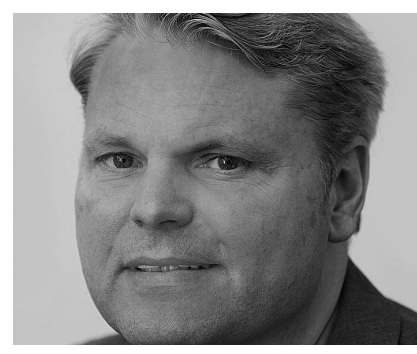

VON DIETMAR WEIDLICH

Rechtsanwalt Dietmar Weidlich berät seit fast zwei Jahrzehnten als Syndikusanwalt Einrichtungen der Sozialwirtschaft bei Neugründungen und Umstrukturierungen sowie Kooperationen und Fusionen. Er hat sich neben dem Gesellschaftsrecht vor allem auf das Vereins- und Stiftungsrecht spezialisiert.

E-Mail

dietmar.weidlich@curacon.de
Viele soziale Organisationen haben in den letzten Jahren einen Aufsichtsrat als Kontrollorgan zur Überwachung der Geschäftsführung eingeführt, wenn sie dazu nicht sowieso schon durch ihre Rechtsform und ihre Größe dazu verpflichtet waren. Die Aufsichtsratmitglieder unterliegen dabei - insbesondere im Innenverhältnis - zahlreichen gesetzlichen Haftungsregelungen.

Die Übernahme eines ehrenamtlichen Aufsichtsratsmandats im Non-Profit-Bereich wurde in der Vergangenheit im Allgemeinen als nicht besonders risikoreich angesehen. Diese Einschätzung beruhte weniger auf einer Analyse der möglichen Haftungsgrundlagen. Vielmehr war es wohl die Annahme anscheinend nicht existierender Präzedenzfälle verbunden mit einer vermeintlichen Schutzsphäre der »Ehrenamtlichkeit ", die die Schlussfolgerung nahe legte, auch bei fehlerhafter Ausübung seines Aufsichtsratsmandats dafür nicht haften zu müssen oder sich in einem haftungsfreien Raum zu bewegen.

Zutreffend war eine solche Annahme jedoch zu keinem Zeitpunkt. Erläutert werden soll die Haftung des fakultativen Aufsichtsrats einer $\mathrm{GmbH}$, da dies bei Non-Profit-Organisationen neben Verein und Stiftung mittlerweile eine der am häufigsten vorkommenden Rechtsformen ist.

Das GmbH-Gesetz verweist in seinem \52 auf zahlreiche Vorschriften des Aktienrechts, so dass sowohl die Vorschriften über den Pflichtenkreis des Aufsichtsrats als auch über die Sorgfaltspflicht und Verantwortlichkeit zur Anwendung kommen, sofern der jeweilige GmbH-Gesellschaftsvertrag nicht deren Anwendung ausschließt.

\section{Haftung im Innenverhältnis}

$\mathrm{Zu}$ unterscheiden ist zwischen der »Innen- oder Regresshaftung « gegenüber der Gesellschaft und der "Außenhaftung " gegenüber Dritten, wobei es sich immer um die Haftung der einzelnen Aufsichtsratsmitglieder handelt, da es keine kollektive Haftung des Aufsichtsrats gibt.
Die Innenhaftung macht den Kernbereich der Aufsichtsratshaftung aus. Als Anspruchsgrundlage dient $\$ 116$ des Aktiengesetzes (AktG), der seinerseits auf $\mathbb{S} 93$ AktG verweist, der die Voraussetzungen der Vorstandshaftung normiert.

Aufsichtsrat und Vorstand unterstehen damit vom Grundsatz her den gleichen Regeln. Eine persönliche Inanspruchnahme durch die Gesellschaft erfordert ein pflichtwidriges und schuldhaftes (Fehl-) Verhalten des Aufsichtsratsmitglieds, das zu einem Schaden des Unternehmens führt ( $\mathbb{S} 116,93$ Abs. 2 AktG). Es haftet also niemand etwa nur dafür, dass er Mitglied des Aufsichtsrats ist.

\section{Pflichtenkreis des Aufsichtrats}

Daher ist es unerlässlich, sich über den Pflichtenkreis des Aufsichtsrats im Klaren zu sein. Ausgangspunkt hierfür ist $\mathbb{} 111$ AktG, der als wesentliche Aufgabe des Aufsichtsrats - die Überwachung der Unternehmensleitung oder Geschäftsführung - definiert. Der konkrete Inhalt der Überwachungspflichten ergibt sich aus der Funktion des Aufsichtsrats und dem ihm vom Gesetz zur Verfügung gestellten Instrumentarium.

Zum Instrumentarium des Aufsichtsrats gehören etwa die Informationsrechte ( $\$ 90 \mathrm{AktG})$, die mit den Berichtspflichten des Vorstands oder der Geschäftsführung korrespondieren, ferner das Einsichts- und Prüfungsrecht ( $\mathbb{S} 111$ Abs. 2 AktG), das Recht zur Einberufung der Gesellschafterversammlung ( $\$ 111$ Abs. 3 AktG), das Recht für den Vorstand oder die Geschäftsführung eine Geschäftsordnung zu erlassen ( $\mathbb{S} 77$ Abs. 2 AktG) einschließlich des Rechts, darin bestimmte Rechtsgeschäfte mit genehmigungspflichtigen $\mathrm{Zu}$ stimmungsvorbehalten zu versehen $(\mathbb{\$} 111$ Abs. 4, Satz 2 AktG).

\section{Rückschauende und begleitend vorausschauende Kontrolle}

In dem sogenannten »ARAG-Urteil « hat der Bundesgerichtshof (BGH) be- 


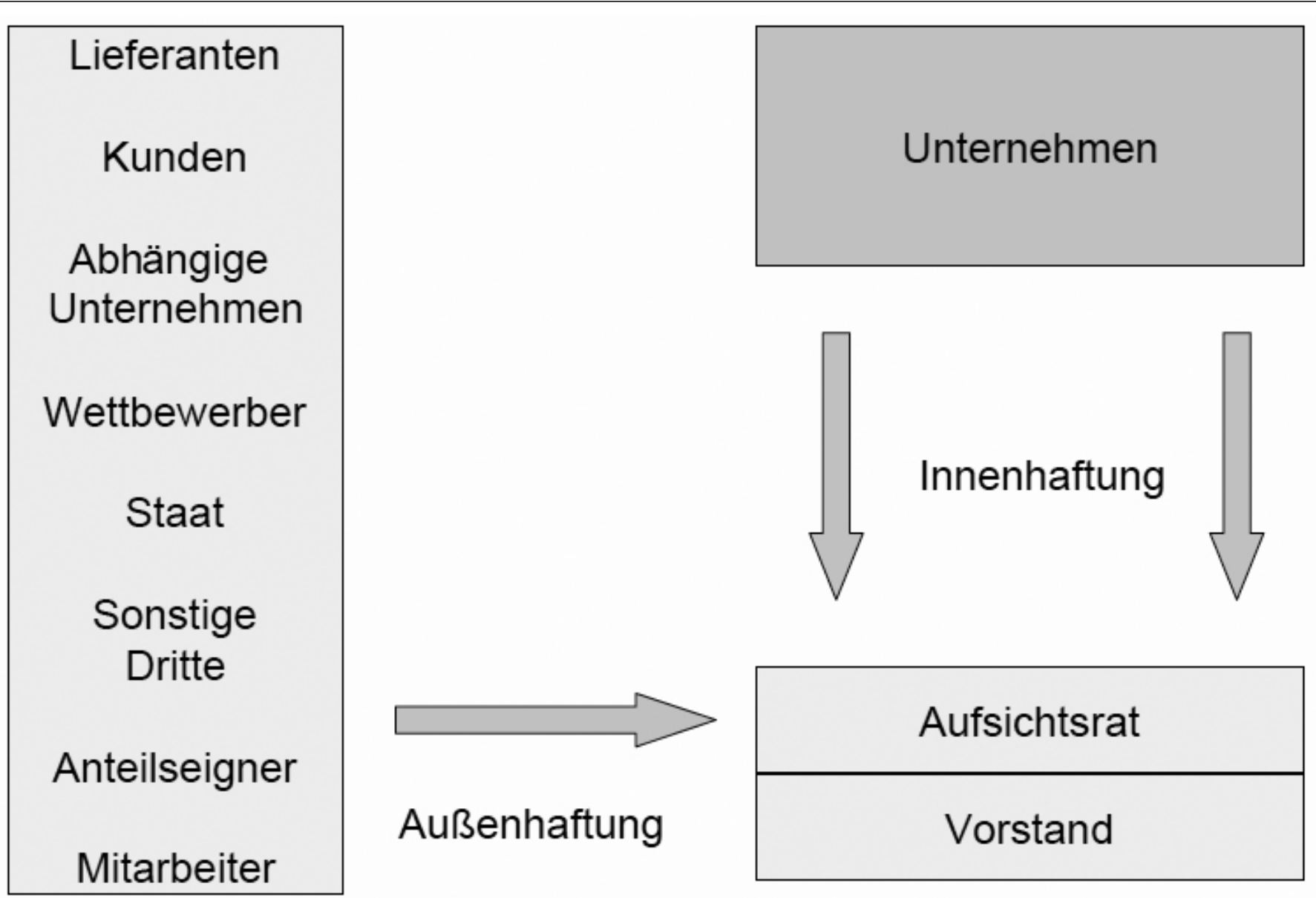

Abb.: Bei der Verantwortung von Aufsichtsratsmitgliedern ist zwischen der Innen- und der Außenhaftung zu unterscheiden.

Quelle: Susanne Koch, Tim Fahje: Ausgewählte aktuelle Fragen aus Rechnungslegung, Wirtschaftsprüfung und Controlling. Schutz vor Inanspruchnahme aus der Haftung als Vorstand und Aufsichtsrat. Internet http://www.uni-hamburg.de/fachbereiche-einrichtungen/fboz/iwp/rut/Praesentation13.ppdf

stätigt, dass zwischen » rückschauender Kontrolle« (z. B. bezogen auf das vergangene Quartal oder Geschäftsjahr) und der sogenannten " präventiven oder begleitend vorausschauenden Kontrolle" der Geschäftsführung zu unterscheiden ist. (1)

Bei der nachträglich rückschauenden Kontrolle geht es um die Ermittlung und Überprüfung abgeschlossener Sachverhalte im Hinblick auf ihre Recht-, Zweck- und Ordnungsmäßigkeit unter Ausnutzung des ihm zur Verfügung stehenden Überwachungsinstrumentariums (s. o.), insbesondere durch sein umfassendes Informationsrecht. Dabei hat der Aufsichtsrat auch zu prüfen, ob der Gesellschaft Ersatzansprüche gegen den oder die Geschäftsführer zustehen. Im Rahmen der nachträglich rückschauenden Kontrolle besteht nur ein sehr eingeschränkter Ermessensspielraum, der gerichtlich voll nachprüfbar ist.

Demgegenüber geht es bei der vorausschauenden Kontrolle um eine unternehmerische Aufgabenstellung, bei der der Aufsichtsrat Einfluss auf die künftige Geschäftspolitik des Unternehmens nimmt. Hierbei kann es sich etwa um Personalentscheidungen handeln (z. B. Berufung, Abbe- rufung und Zusammensetzung der Geschäftsführung), die Mitwirkung bei der strategischen Planung oder die Zustimmung zu bedeutsamen Einzelentscheidungen. Insoweit steht dem Aufsichtsrat - wie das »ARAG-Urteil « ausführt (2) - ein unternehmerischer Gestaltungsspielraum zu, der gerichtlich nur sehr beschränkt überprüfbar ist.

\section{Vorliegen einer Pflichtverletzung}

Pflichtverletzungen im Rahmen der nachträglich rückschauenden Kontrolle kommen immer dann in Betracht, wenn entweder das zur Verfügung stehende Überwachungsinstrumentarium nicht oder nur unvollkommen genutzt wird oder die sich aus der Überprüfung der Sachverhalte ergebenden Konsequenzen nicht gezogen werden. Bei ersterem dürfte der Verzicht auf detaillierte Informationen im Vordergrund stehen (z. B. es werden zu wenige oder gar keine Aufsichtsratssitzungen abgehalten, Berichte der Geschäftsführung werden nur überflogen oder bleiben ohne Nachfrage, beabsichtigte Maßnahmen oder kritische Geschäfte werden nicht hinterfragt).
Haftungsbegründend dürften sich aber vor allem nicht gezogene Konsequenzen auswirken (z. B: Ablehnung einer erforderlichen Zustimmung, Aufforderung zu Kurskorrekturen und als letzte Konsequenz die unterlassene Abberufung eines oder mehrerer Geschäftsführer. Mit einem derartigen Gewährenlassen verletzt der Aufsichtsrat eindeutig seine Kontrollpflichten. Dies gilt insbesondere in Krisensituationen, in denen die Kontrolldichte noch zunimmt. (3)

Kommt der Aufsichtsrat im Rahmen seiner Prüfung zu dem Ergebnis, dass der Gesellschaft Ersatzansprüche gegen $\mathrm{Ge}$ schäftsführer zustehen und unterlässt er es, diese geltend zu machen, macht er sich grundsätzlich selbst schadensersatzpflichtig. Lediglich in ganz besonders gelagerten Ausnahmefällen können übergeordnete Unternehmensinteressen gegen eine solche Unterlassung rechtfertigen.

Weniger risikobehaftet ist der Bereich der »vorausschauenden Kontrolle «. Soweit sich die Entscheidungen des Aufsichtsrats innerhalb seines " unternehmerischen Gestaltungsspielraums « halten, liegt keine Pflichtverletzung vor. Der Gestal- 
tungsspielraum wird jedoch überschritten, wenn gegen gesetzliche oder gesellschaftsvertragliche Regelungen verstoßen wird.

\section{Verschulden}

Das Aufsichtsratsmitglied muss die Pflichtverletzung schuldhaft verursacht haben. Der Verschuldensmaßstab folgt aus $\mathbb{9} 93$ (für den Vorstand), der über $\$ 116$ AktG für Aufsichtsratsmitglieder die "Sorgfaltspflicht eines ordentlichen und gewissenhaften Überwachers und Beraters « (4) verlangt. sichtsrats in der Praxis keine große Rolle. Zudem schützt $\mathbb{} 31$ BGB analog die Aufsichtsratsmitglieder vor den Ansprüchen Dritter, wenn die Aufsichtsratsmitglieder den Schaden im Rahmen ihrer Aufsichtsratstätigkeit verursacht haben. Lediglich im Falle einer Insolvenz des Unternehmens kann ein geschädigter Dritter, der aufgrund der Insolvenz keinen Schadensersatz vom Unternehmen erlangen kann, gemäß $\mathbb{} \int 93$ Abs. 5 AktG analog gegen Aufsichtsratsmitglieder bei zumindest grob fahrlässiger Pflichtverletzung den Anspruch geltend machen, der eigentlich der Gesellschaft im Innenverhältnis (s. o.) zustünde.

\section{"Aufsichtsratsmitglieder müssen die ,Sorgfalts- pflicht eines ordentlichen und gewissenhaften Überwachers und Beratersı an den Tag legen«}

Grundsätzlich haften Aufsichtsratsmitglieder auch für leichte Fahrlässigkeit, sofern die Haftung z. B. im Gesellschaftsvertrag nicht Vorsatz und grobe Fahrlässigkeit beschränkt ist.

\section{Beweislastumkehr}

Die Besonderheit des $\$ 93$ AktG besteht aber in der in Abs. 2 Satz 2 angeordneten Beweislastumkehr. Das bedeutet, dass die Gesellschaft lediglich das schadensstiftende Verhalten benennen muss, während sich das Aufsichtsratsmitglied vom Vorwurf der Pflichtwidrigkeit einschließlich des Verschuldens zu entlasten hat. Der Entlastungsbeweis kann beispielsweise darin bestehen, dass das betreffende Mitglied nachweisen kann, am Zustandekommen des fraglichen Aufsichtsratsbeschlusses entweder überhaupt nicht beteiligt gewesen zu sein (z. B. keine Teilnahme an der Sitzung) oder laut Protokoll ausdrücklich dagegen gestimmt zu haben.

Können die Aufsichtsratsmitglieder diesen Entlastungsbeweis nicht führen, haften alle Aufsichtsratsmitglieder - bis zum Beweis des Gegenteils - gesamtschuldnerisch für den Schaden, der der Gesellschaft z. B. aus der unterlassenen Abberufung oder der unterlassenen Geltendmachung von Schadensersatzansprüchen gegen Geschäftsführer entstanden ist.

\section{Haftung im Außenverhältnis}

Im Gegensatz zur Haftung des Geschäftsführers, der ja die Gesellschaft im Außenverhältnis als gesetzlicher Vertreter vertritt, spielt die Außenhaftung des Auf-
Schadensersatzansprüche gegen Aufsichtsratsmitglieder verjähren gemäß $\mathbb{5} 52$ Abs. $3 \mathrm{GmbHG}$ in fünf Jahren.

\section{Resümee}

Die Relevanz der Aufsichtsratshaftung im Non-Profit-Bereich wird bis heute immer noch unterschätzt, da bestehende Ersatzansprüche der Gesellschaft in der Vergangenheit häufig wegen Interessenkonflikten oder aus Imagegesichtspunkten nicht durchgesetzt wurden. Dies dürfte sich bei erheblich knapper werdenden Mitteln und einer steigenden Zahl an Insolvenzen auch in der Sozialwirtschaft künftig ändern.

\section{Anmerkungen}

(1) BGHZ 135, 244, 245 f.; so auch schon BGHZ 114, 127, 130.

(2) BGHZ 135, 244, 254, 255.

(3) Potthoff/Trescher, Das Aufsichtratsmitglied, 4. Aufl., S. 197 f.

(4) BGH WM 1983, 10, BGHZ 85, 293, 295.

\section{Partnerschaft von Sozialdiensten mit Bürgern}

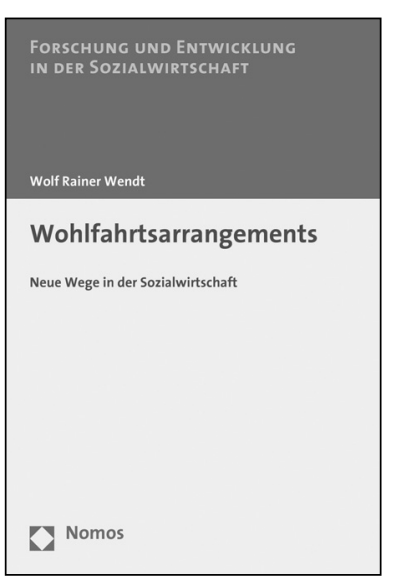

Wohlfahrtsarrangements Neue Wege in der Sozialwirtschaft

Von Prof. Dr. Wolf Rainer Wendt

2010, 132 S., brosch., 24,- $€$, ISBN 978-3-8329-5244-O

(Forschung und Entwicklung in der Sozialwirtschaft, Bd. 6)

Sozialdienste kooperieren mit ihren Nutzern. Der Band erörtert Arrangements, in denen Versorgungsangebote auf besondere, sich verändernde Bedarfslagen zugeschnitten werden. Innovative Formen der Wohlfahrtsproduktion werden in theoretischen Beiträgen sowie anhand empirischer Beispiele aus der Jugend- und Sozialhilfe untersucht.

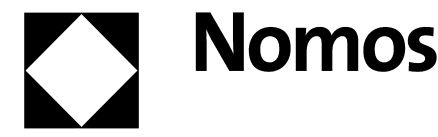

